\title{
An embedded fracture modeling framework for simulation of hydraulic fracturing and shear stimulation
}

\author{
Jack H. Norbeck ${ }^{1} \cdot$ Mark W. McClure ${ }^{3}$ Jonathan W. Lo ${ }^{2} \cdot$ Roland N. Horne ${ }^{1}$
}

Received: 26 March 2015 / Accepted: 20 October 2015 / Published online: 13 November 2015

(C) The Author(s) 2015. This article is published with open access at Springerlink.com

\begin{abstract}
A numerical modeling framework is described that is able to calculate the coupled processes of fluid flow, geomechanics, and rock failure for application to general engineering problems related to reservoir stimulation, including hydraulic fracturing and shear stimulation. The numerical formulation employs the use of an embedded fracture modeling approach, which provides several advantages over more traditional methods in terms of computational complexity and efficiency. Specifically, the embedded fracture modeling strategy avoids the usual requirement that the discretization of the fracture domain conforms to the discretization of the rock volume surrounding the fractures. As fluid is exchanged between the two domains, conservation of mass is guaranteed through a coupling term that appears as a simple source term in the governing mass balance equations. In this manner, as new tensile fractures nucleate and propagate subject to mechanical effects, numerical complexities associated with the introduction of new fracture control volumes are largely negated. In addition, the ability to discretize the fractures and surrounding rock volume independently provides the freedom to choose an acceptable level of discretization for each domain separately. Three
\end{abstract}

Jack H. Norbeck

jnorbeck@stanford.edu

1 Department of Energy Resources Engineering, Stanford University, Stanford, CA 94305, USA

2 Petroleum and Geosystems Engineering, The University of Texas at Austin, Austin, TX 78712, USA

3 McClure Geomechanics LLC, Palo Alto, CA 94301, USA numerical examples were performed to demonstrate the utility of the embedded fracture model for application to problems involving fluid flow, mechanical deformation, and rock failure. The results of the numerical examples confirm that the embedded fracture model was able to capture accurately the complex and nonlinear evolution of reservoir permeability as new fractures propagate through the reservoir and as fractures fail in shear.

Keywords Embedded fracture modeling - Reservoir geomechanics $\cdot$ Hydraulic fracture $\cdot$ Shear stimulation . Fractured reservoir simulation

Mathematics Subject Classification (2010) 65N08 . 74F10 $\cdot 90-08 \cdot 90 \mathrm{C} 30 \cdot 90 \mathrm{C} 90$

\section{Introduction}

In many reservoir engineering applications, it is imperative to incorporate a realistic description of the geologic structure of the reservoir into conceptual models and numerical models in order to establish appropriate interpretations of reservoir behavior. Several examples include hydraulic fracture treatment design, interpretation of microseismic monitoring data, and development of reservoir management strategies related to induced seismicity. In each of these cases, the interaction between fluid flow and the geomechanical response of fractured and faulted rock will have a direct influence on the reservoir behavior, and therefore also on the engineering decisions that must be made.

In geologic settings where fractures and faults are expected to have first-order impacts in terms of flow behavior, it is important to recognize that the reservoir systems are 
mechanically active. During development and operation of a resource, local-scale and reservoir-scale permeability and storativity can evolve as fractures deform and fail in shear, or as intact rock fails in tension. The local state of stress throughout the reservoir controls the manner in which the permeability and storativity changes manifest. It is necessary to make use of numerical modeling to investigate these types of reservoir processes for practical applications, but many traditional reservoir models neglect geomechanical processes or are based upon a set of limiting assumptions that obviate the influence of significant physical mechanisms.

In this paper, we introduce an efficient numerical modeling framework that is able to model the coupled physical processes of fluid flow and mechanical deformation of fractures, faults, and surrounding matrix rock. The framework is able to incorporate an explicit representation of the geologic structure of the reservoir by using an embedded fracture modeling (EFM) strategy [21]. The EFM approach is an extension of more traditional finite-volume-based discrete fracture models (DFM), suggesting relatively straightforward integration with industry-standard reservoir simulators. A fracture mechanics-based approach to mechanical modeling allows for accurate calculation of the complex stress distributions that arise near fracture tips, so fracture propagation problems are approached in a rigorous manner. Detailed models of friction evolution along fracture and fault surfaces are included in order to model shear failure and seismicity. To accommodate different types of rock, the model is flexible enough to incorporate a range of constitutive relationships necessary to describe permeability and storativity evolution of fracture networks due to changes in effective stress and shear failure.

The integration of the EFM approach into a geomechanical and fracture propagation model is the principal achievement of the present work. The embedded fracture approach is a numerical method that provides the critical translation necessary to attack problems that would otherwise be intractable from a computational standpoint. The key element of the EFM formulation is the treatment of the fracture system and the surrounding matrix rock volume as two separate computational domains $[19,21]$. This allows for the two domains to be discretized completely independently, negating the cumbersome requirement of a matrix discretization that must conform to the fracture discretization associated with traditional discrete fracture models.

In the EFM discretization, conservation of mass is enforced as fluid is exchanged between the two domains through the application of physics-derived coupling terms that appear as source terms in the continuity equations. In this manner, tensile fractures that nucleate and propagate subject to geomechanical considerations can be incorporated into the numerical model during a simulation with negligible amount of computational overhead.

Previous authors have applied EFM to investigate geomechanical effects in fractured reservoirs, but this work has been limited in scope to simplified models that embody the geomechanics into empirical relationships $[16,26]$. In this work, the reservoir model introduced by McClure [23] and McClure and Horne [24] was extended to incorporate the EFM strategy in order to combine the effects of matrix-fracture mass exchange and a rigorous treatment of geomechanics under a unified framework. The fluid flow and geomechanical calculations are performed in a coupled sequential-implicit manner. Fracture propagation is permitted, and is based upon evaluating the mode-I stress intensity factor near fracture tips. Shear failure of preexisting fractures is permitted subject to a modified Mohr-Coulomb criterion [13]. The mechanical interaction between fractures as they deform is included in the model. Reservoir-scale permeability evolution emerges as a result of deformation of individual fracture and fault planes, shear failure on preexisting fractures and faults, and propagation of tensile fractures. Limitations of the model arise from the following important assumptions: the mechanical properties of the rock are homogeneous and constant, elastic deformation is quasistatic, poroelastic and thermoelastic deformations are neglected, and the domain is two-dimensional (2-D) so all fractures in the model have the same out-of-plane dimension.

The primary purpose of this work was to verify the accuracy of the EFM approach for applications in which geomechanical effects have a first-order impact on reservoir behavior. The embedded fracture-based model developed in this work was compared to a traditional DFM for three different numerical examples of practical interest. When appropriate, the numerical results were also compared against analytical or semianalytical solutions. In addition, the accuracy of two extremely efficient approximate models, a one-dimensional (1-D) leakoff model and a zero leakoff model, were also compared in order to help quantify their range of practical applicability for reservoir stimulation modeling.

The remainder of this paper is organized as follows. In Section 2, the numerical formulation for the EFM framework is presented. The traditional DFM, 1-D leakoff approximation model, and zero leakoff approximation model that were used for comparison are also discussed. In Section 3, the major components in the geomechanical module are described. In Section 4, we demonstrate that the models achieved good agreement with an analytical solution for a problem involving injection into an infiniteconductivity fracture. Next, we modeled a shear stimulation treatment of a relatively complex fracture network, and the results are presented in Section 5. Significant nonlinear effects due to shear slip-enhanced fracture permeability 
made this a challenging numerical problem. Finally, the utility of the EFM framework was highlighted by modeling mode-I fracture propagation of a vertical, two-wing fracture. The results of the fracture propagation study are shown in Section 6. In Section 7, several practical applications for the present model are illustrated, and concluding remarks are discussed.

\section{Fluid flow module}

The reservoir model introduced by McClure [23] and McClure and Horne [24] was developed originally to model reservoir stimulation treatments in low-permeability settings, such as hydraulic fracturing in shale gas reservoirs or shear stimulation in geothermal reservoirs. The model assumed that intrinsic matrix permeability in these settings is usually low enough to justify neglecting mass transfer between the fracture systems and surrounding matrix rock. In the present work, this model was extended to include the effects of matrix-fracture mass transfer. An EFM strategy was adopted in order to overcome several severe numerical and practical limitations of more traditional DFM approaches for application to reservoir stimulation problems.

In this section, the numerical formulation for the embedded fracture model is described in detail. In the sections that follow, we describe studies where we compared the EFM approach with a DFM approach that had been implemented previously in the McClure [23] model. Here, the details of the DFM model are described for the reader's reference. In addition, the EFM and DFM models were compared with two relatively computationally efficient approximate models, namely the 1-D leakoff approximation model and the zero leakoff approximation model. The details of these models are described briefly.

\subsection{Embedded fracture model description}

In traditional reservoir simulation, fractured reservoirs are commonly modeled using the double porosity model [17, 42]. This model is applicable if the fracture orientations and lengths are relatively randomly distributed and the fracture system is connected extensively. More importantly, application of the double porosity model typically assumes that the properties and geometry of the fracture network remain relatively constant.

In order to honor more realistic representations of fractured reservoir geology, discrete fracture approaches have been developed. For example, Karimi-Fard et al. [14] presented a DFM in which the geometry of the fractures and faults are captured by discretizing them explicitly in lowerdimensional space, and creating a matrix discretization that conforms to the fractures. In general, DFM approaches are useful in settings where production is dominated by flow through fractures (e.g., formations with low matrix permeability) or if fractures tend to have preferred orientations. However, traditional DFM techniques are subject to several well-documented drawbacks. Most notable is that a matrix discretization that conforms to the fractures inevitably results in a large number of "small" matrix control volumes in areas where there are many fractures or where fractures intersect at low angles. In some cases, the geologic structure of the reservoir must be sacrificed for numerical convenience.

Moreover, DFM techniques are not well suited for fracture propagation problems in which the fracture networks are growing over time. Previous work has been done in the area of developing models that apply adaptive grid refinement as fractures propagate $[12,33]$. This requires a significant level of computational overhead, and the numerical results have been observed to be grid-dependent. Alternatively, it is possible to define planes where hydraulic fractures may potentially propagate in advance of a simulation, and then prediscretize the system around these potentially forming planes. Naturally, this approach will require an unnecessarily high number of additional degrees of freedom, and, perhaps worse, involves making implicit assumptions about the mechanics of fracture propagation.

\subsubsection{Embedded fracture model overview}

In the present work, the use of traditional DFM techniques was avoided, and instead the EFM approach was adopted. In the EFM approach, the fracture and matrix domains are treated as separate computational domains. The two systems are discretized completely independently (i.e., a conforming mesh is not required; see Fig. 1), and mass conservation is strictly enforced through physics-derived coupling terms. In fact, EFM is conceptually very similar

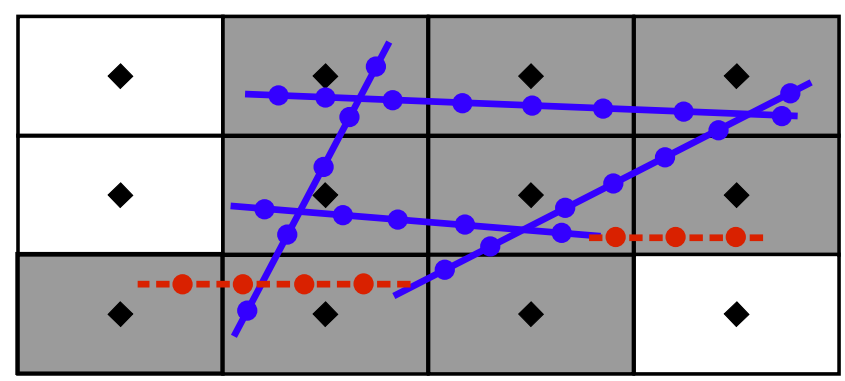

Fig. 1 Schematic of the embedded fracture discretization strategy. The solid blue lines are natural fractures, and the dashed red lines are hydraulic fractures. The circles represent the centers of fracture control volumes, and the diamonds represent the centers of matrix control volumes. The matrix control volumes that will pick up EFM coupling terms are shaded gray 
to dual porosity or dual permeability models, but is able to maintain a more realistic representation of complex geologic features. As will be demonstrated in the numerical examples that follow, the ability to define realistic representations of the geologic structure of a reservoir and the use of a nonconforming grid are the critical features that make the EFM approach an attractive modeling strategy to perform rigorous geomechanical analyses.

The EFM approach was introduced originally by Lee et al. [19], and later expanded upon by $\mathrm{Li}$ and Lee [21]. Karvounis [15] developed a heat and mass transfer geothermal model based on EFM, and demonstrated that EFM can obtain a suitable degree of accuracy with improved computational performance compared to traditional models. Hajibeygi et al. [9] incorporated EFM into an iterative multiscale finite volume scheme. Several studies have compared DFM to EFM for multiphase flow problems and demonstrated that EFM was able to capture a high degree of accuracy at a reduced computational expense [25, 32]. Ding et al. [4] drew upon EFM fundamentals and calculated the matrix-fracture transmissibility numerically to be able to capture pressure transients in the near-fracture region more accurately.

Recently, EFM has also been used in geomechanics applications. Moinfar et al. [26] incorporated a treatment for calculating fracture permeability evolution due to changes in effective stress within an EFM framework, but did not include a formal treatment for geomechanics. Karvounis et al. [16] extended their model to include a proxy geomechanical model based on changes in pore pressure to investigate injection-induced seismicity. Norbeck et al. [27] and Norbeck and Horne [28] introduced an EFM-based model that integrated a rigorous treatment of fluid flow, fractured reservoir mechanics, and fracture propagation. Norbeck and Horne [29] performed a study of porothermoelastic effects on injection-induced seismicity using a rate-and-state earthquake model.

It should be noted that in some of the works cited previously, the concept of EFM was applied in a context related to upscaling techniques $[9,21]$. In those applications, it was assumed that fractures that existed at a relatively small scale could be homogenized in order to obtain effective "damaged matrix rock" properties, and the geometries of the larger fracture systems expected to contribute to flow at a reservoir scale were maintained explicitly. For the purposes of this paper, it is sufficient to recognize this distinction purely at the conceptual level. In the remainder of this paper, it is assumed that any reference to matrix permeability may imply an effective upscaled permeability, and any fracture domain is representative of a scale of practical engineering interest.

\subsubsection{Numerical formulation of the embedded fracture model}

The key insight introduced by Li and Lee [21], that the fracture and matrix domains can be discretized independently, is leveraged by expressing the mass conservation equations for the matrix and fracture domains separately. For a porous medium saturated with single-phase fluid, the continuity equations can be written, for flow in the matrix domain, as:

$\nabla \cdot\left(\rho \lambda \mathbf{k}^{m} \nabla p^{m}\right)+\tilde{m}^{w m}+\widetilde{\Psi}^{f m}=\frac{\partial}{\partial t}(\rho \phi)$,

and, for flow in the fracture domain, as:

$\nabla \cdot\left(\rho \lambda e \mathbf{k}^{f} \nabla p^{f}\right)+\widetilde{m}^{w f}+\widetilde{\Psi}^{m f}=\frac{\partial}{\partial t}(\rho E)$.

Here, $p^{m}$ is fluid pressure in the matrix domain, $p^{f}$ is fluid pressure in the fracture domain, $\rho$ is fluid density, $\lambda$ is inverse of fluid viscosity, $\mathbf{k}^{m}$ is the diagonal matrix permeability tensor, $\mathbf{k}^{f}$ is the diagonal fracture permeability tensor, $\phi$ is matrix porosity, $e$ is fracture hydraulic aperture, $E$ is fracture void aperture, $\widetilde{m}^{w m}$ is a normalized mass source term related to wells in the matrix domain, and $\widetilde{m}^{w f}$ is a normalized mass source term related to wells in the fracture domain. In addition to the usual terms related to flux, wells, and storage, the terms $\widetilde{\Psi}^{f m}$ and $\widetilde{\Psi}^{m f}$ are introduced to account for mass transfer between the two domains. To ensure continuity upon integration over the respective control volumes, these mass transfer terms take the following form [9]:

$\widetilde{\Psi}^{f m}=\Upsilon\left(p^{f}-p^{m}\right) / V$,

and

$\widetilde{\Psi}^{m f}=\Upsilon\left(p^{m}-p^{f}\right) / A$,

where the parameter $\Upsilon$ is a transmissibility called the fracture index and is analogous to the Peaceman well index [31]. The normalization parameters in Eqs. 3 and 4 are $V$, the bulk volume of the matrix control volume, and $A$, the surface area of the fracture control volume. Comparing terms in Eqs. 1-4, the units indicate that the fracture mass balance equations are expressed on a lower-dimensional manifold.

Similar to the treatment of wells in traditional reservoir simulators, the fracture index serves to capture subgrid behavior of the pressure gradient near fractures. In this work, the derivation provided by $\mathrm{Li}$ and Lee [21] was followed to calculate the fracture index. The assumptions in the derivation are as follows: (a) flow in the vicinity of the fractures is linear, (b) the fractures fully penetrate the matrix 
control volume in the out-of-plane direction, and (c) the matrix and fracture pressures represent average pressures over their respective control volumes.

The rate of mass transfer from a fracture control volume into a matrix control volume is defined as:

$\Psi^{f m}=\Upsilon\left(p^{f}-p^{m}\right)$.

This term has units of mass per time. Assuming that flow is linear (i.e., 1-D) in the local region near the fracture, the mass exchange rate can be described alternatively by integrating the Darcy flux over the surface area of the fracture:

$\Psi^{f m}=A^{f} \rho \lambda k^{*} \nabla p \cdot \mathbf{n}$,

where $A^{f}$ is the total fracture surface area (i.e., both faces of the fracture), $k^{*}$ is an effective fracture-normal permeability, $\mathbf{n}$ is the unit normal vector to the fracture face, and the pressure gradient term is:

$\nabla p=\frac{p^{f}-p^{m}}{L} \mathbf{n}$.

Here, $L$ represents the average normal distance from the fracture surface in the matrix control volume, which can be calculated numerically for complex fracture and matrix control volume geometries [9]. The effective permeability, $k^{*}$, represents the ability for fluid to flow in the direction perpendicular to the fracture. In reservoir settings with low matrix permeability and highly conductive fractures, $k^{*}$ will likely be dominated by the matrix rock permeability. In geologic settings where relatively thick faults with sealing properties exist, the fracture (fault) permeability can affect $k^{*}$ significantly. In general, $k^{*}$ can be considered a harmonic mean of the matrix and fracture permeabilities:

$\frac{L}{k^{*}}=\frac{L}{k_{\perp}^{m}}+\frac{W}{k_{\perp}^{f}}$,

where $k_{\perp}^{m}$ and $k_{\perp}^{f}$ are the matrix and fracture permeabilities, respectively, resolved in the direction normal to the fracture, and $W$ is the physical half-width of the fracture. In Eq. 8, it was assumed that $L \gg W$.

Equating the right-hand side expressions in Eqs. 5 and 6 allows for the determination of the fracture index:

$\Upsilon=I \rho \lambda k^{*}$

where $I$ is a grid dependent property with units of length that can be calculated as:

$I=\frac{A^{f}}{L}$.

With the matrix-fracture mass flux terms that appear in Eqs. 1 and 2 now fully defined, the utility of the EFM approach for problems that involve fracture propagation is revealed. The coupling between the fracture and matrix domains has been reduced to a collection of simple source terms. Numerical complexities associated with conforming mesh approaches that would tend to make fracture propagation problems become intractable for problems with many fractures are now avoided with EFM. Standard finite volume schemes can be used to discretize Eqs. 1 and 2 $[1,14]$.

\subsection{Discrete fracture model description}

The DFM was implemented using the finite volume method and a conforming mesh of the rock volume around the fractures. The volume around the fractures was discretized with triangular control volumes, aided by the program Triangle [35]. Triangle is an algorithm designed to create Delaunay triangularizations of 2-D regions. The finite volume method was implemented according to the method described by Karimi-Fard et al. [14].

An important problem is that Delaunay triangularization does not guarantee uniform or smoothly varying linesegment length along domain edges, which in this case are the fracture elements. This can create problems in the boundary element mechanical calculations described in Section 3, which are inaccurate unless fracture element length is uniform or gradually varying. Therefore, Triangle could not be used to generate a true Delaunay triangularization.

To guarantee uniform fracture element size, the fractures were discretized first by imposing a constant element length (with some minor and unavoidable deviation from constant length at fracture tips and intersections). Next, a uniform grid of matrix nodes was superimposed over the fracture network. Third, nodes were identified that were in close proximity to fracture elements, and they were removed. Finally, the list of fracture elements and matrix nodes was provided to Triangle, which was used to produce a constrained Delaunay triangularization. The triangularization was "constrained" in the sense that the algorithm was required to use only the fracture elements and matrix nodes provided and was not permitted to subdivide the fracture elements. Because of the constraint, the mesh was not guaranteed to be truly Delaunay, which degraded the quality of the mesh and the accuracy of the calculations of flow in the matrix. Despite this problem, the approach was used because it was more important to avoid inaccuracy in the mechanical calculations due to unevenly sized fracture elements than inaccuracy in matrix flow calculations due to high aspect ratio triangles. It should be noted that this entire issue is avoided with the EFM approach, which does not require a conforming mesh between the matrix and fracture 
elements. Figure 2 is an example of the type of conforming mesh used in the calculations.

\subsection{One-dimensional leakoff approximation model description}

For a well connected to a highly conductive fracture within an infinite reservoir, flow near the fracture has been shown to be linear at early times [8]. This suggests that a useful approximation to model the leakoff behavior near fractures is to assume 1-D flow away from the fracture. In this work, the semianalytical method of Vinsome and Westerveld [41] was used to develop an approximate model that is relatively efficient computationally compared to the EFM and DFM approaches. The purpose of the simplified model is to avoid numerical discretization of the volume of rock surrounding the fractures, while still accounting for fluid exchange between the two domains.

The model treats fluid leakoff at each fracture control volume using a sink term that is independent from all other fracture control volumes. The key advantage of the Vinsome and Westerveld [41] method is that it gives a highly accurate and efficient solution to the diffusivity equation in 1-D, even for arbitrarily varying pressure in the fracture. In contrast, the Carter leakoff model assumes constant pressure in the fracture [11], a simplifying assumption that seriously reduces model generality.

The Vinsome and Westerveld [41] method was created originally as a model of heat loss due to conduction into cap rock. However, the equation for heat conduction is identical to the equation for single-phase fluid flow in a porous media with constant pore and fluid compressibilities, matrix permeability, and fluid viscosity. Therefore, the method can be adapted easily by changing the variables in the original equations of Vinsome and Westerveld [41] to their equivalents for flow in porous media, assuming that the aforementioned variables are considered as constants.

The assumptions of 1-D leakoff and no interference between fractures are justified if the fracture spacing is sufficiently large relative to the penetration distance of the pressure signal and if the injection duration is short enough to preclude a change in the flow regime (e.g., towards late-time radial flow). The penetration distance, $l$, can be estimated as [2]:

$l=4 \sqrt{\frac{k^{m}}{\mu \phi_{0} c}}$,

where the total compressibility, $c$, is the sum of the pore and fluid compressibilities, $\mu$ is fluid viscosity, and $\phi_{0}$ is the initial porosity of the matrix rock. If fractures are in pressure communication with other nearby fractures, then the 1-D leakoff approximation tends to overestimate the amount of leakoff. Relatively high leakoff suppresses pressure within the fracture domain, which discourages shear failure and fracture propagation.

\subsection{Zero leakoff approximation model description}

In geologic settings where the intrinsic permeability of the matrix rock is extremely low, a useful approximation is that
Fig. 2 Example of a triangular discretization used for the conforming mesh discrete fracture model. The red lines are the natural fractures, and the blue lines are the edges of the triangular matrix control volumes

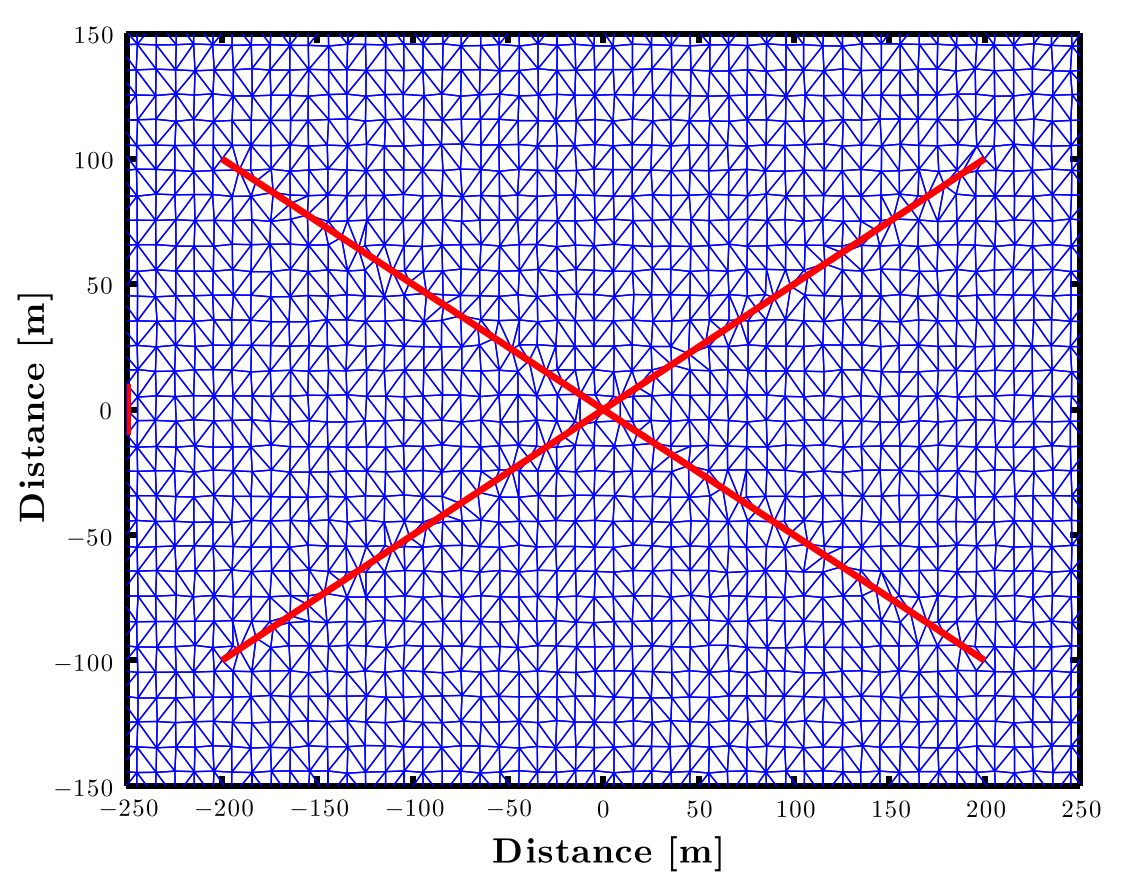


the matrix rock is impermeable. In this case, fluid flow can occur only within a network of connected fractures, and no fluid is able to leakoff into the surrounding rock. Under this assumption, there is no flow in the matrix rock, and the volume surrounding the fractures does not require discretization. The improvement in computational efficiency that can be achieved by avoiding discretization of the matrix rock volume can be tremendous.

This approximation represents a lower bound on the amount of leakoff that would occur during a stimulation treatment. Neglecting leakoff tends to promote elevated pressure in the fracture domain, which encourages both shear stimulation and fracture propagation. Even in scenarios in which the implicit assumptions of this approximation are not strictly valid, the model can be used to provide informative constraints on reservoir behavior.

\section{Geomechanics module}

The present numerical model was built upon the framework introduced by McClure [23] for coupling fluid flow in fractures and fracture deformation. The reader is referred to McClure [23] and McClure and Horne [24], where thorough descriptions of the assumptions and the numerical formulation for the geomechanics module are explained. Here, we provide a brief overview of the major components of the model.

\subsection{Fracture deformation}

We are interested principally in modeling systems that contain a large number of fractures and faults, and therefore the displacement fields that arise as a result of fracture deformation are expected to be discontinuous. A boundary element method called the displacement discontinuity (DD) method is capable of calculating the complex displacement fields due to the mechanical interaction between fractures as they deform [3]. In addition, the DD method has been demonstrated to calculate accurate stress distributions in the vicinity of fracture tips, which is necessary to model fracture propagation [30].

The DD model assumes a 2-D faulted and fractured porous domain, saturated with a single-phase fluid. The mechanical properties of the intact matrix rock are homogeneous. Deformations occur quasistatically, and linear elasticity applies. Using the approach described by Shou and Crouch [37], a linear system of equations can be developed that relates the mode-I (opening) and mode-II (sliding) fracture deformations to the traction boundary conditions along the fractures. The primary variables that must be solved for are the mode-I and mode-II displacement discontinuities, $\Delta e$ (or equivalently $\Delta E$ ) and $\Delta \delta$, respectively.
The traction boundary conditions are functions of the effective normal stress distributions along the fractures. The effective normal stress, $\bar{\sigma}$, at a specific location along a fracture surface is defined as:

$\bar{\sigma}=\sigma^{R}+\Phi-p^{f}$,

where $\sigma^{R}$ is the normal component of the remote tectonic stress, and $\Phi$ is the normal component of the elastic stress transfer that occurs as nearby fracture elements deform. Compressive stress has been taken as positive in this sign convention. The shear stress, $\tau$, acting on a fracture surface is:

$\tau=\tau^{R}+\Theta-\eta v$,

where $\tau^{R}$ is the shear component of the remote tectonic stress, $\Theta$ is the shear component of the elastic stress transfer that occurs as nearby fracture elements deform, $\eta$ is a radiation damping coefficient, and $v$ is sliding velocity. The radiation damping term is used to approximate inertial effects when sliding occurs very rapidly [34]. In the model, $\Phi$ and $\Theta$ are calculated using the DD method. A Mohr-Coulomb shear failure criterion is used for the fracture sliding calculations, whereby the frictional strength of a fracture, $\tau_{s}$, is defined as [13]:

$\tau_{s}=f \bar{\sigma}+S$.

Here, $f$ is the coefficient of friction, and $S$ is the fracture cohesion.

Fractures can be classified into several groups depending on their local state of stress. A fracture element is classified as closed if it is bearing compression such that its walls are in physical contact. The walls of closed fractures are rough surfaces, and so these fractures have the ability to transmit and store fluid. Elastic stress transfer due to mode-I deformations of closed fractures is assumed to be negligible. A fracture element is open if the effective stress drops to zero such that the fracture walls become out of contact. Open fractures deform subject to fracture mechanics considerations. If the shear stress acting on the fracture is less than its frictional resistance to slip (i.e., $\tau<\tau_{s}$ ), then the fracture is classified as stuck. Mode-II deformations and the associated elastic stress transfer is assumed to be negligible for stuck fractures. If the shear stress acting on the fracture becomes equal to its frictional resistance to slip (i.e., $\tau=\tau_{s}$ ), the failure criterion has been met and the fracture is classified as sliding.

The boundary conditions that must be enforced for the mechanical deformation problem depend on these classifications. For closed fractures that are sliding, the following boundary condition is enforced:

$\left|\tau^{R}+\Theta-\eta v\right|=f \bar{\sigma}+S$ 
For open fractures, the following boundary conditions are enforced:

$\bar{\sigma}=0$,

and

$\left|\tau^{R}+\Theta-\eta v\right|=0$.

Equations 1, 2, and 15-17 can be recast in residual form to develop a coupled system of nonlinear equations. The primary variables involved in these equations are $p^{m}, p^{f}$, $\Delta e$, and $\Delta \delta$. In the model, a sequential implicit strategy is used to solve the fully coupled system of equations [18]. The equations involving fluid pressure and opening displacement are grouped together and solved simultaneously $(1,2$, and 16), and the residual equations involving shear displacement are solved separately (15 and 17). The sequential strategy iterates between these two groups until all residual equations have converged to within a prescribed tolerance.

\subsection{Fracture permeability evolution}

An important component of the model used in this work is the ability for the permeability of individual fractures to evolve through time. Fracture permeability depends the local state of stress throughout the reservoir and on failure processes [44]. In our model, the transmissivity of a fracture is assumed to behave according to Poiseuille's law for flow between two parallel plates, which suggests that the fracture-parallel transmissivity, $T^{f}$, is related to the hydraulic aperture of the fracture [38]:

$T^{f}=e k_{\|}^{f}=\frac{e^{3}}{12}$.

Laboratory experiments have shown that for some types of rock, shear slip can lead to a self-propping behavior that can result in altered permeability [20]. An empirical model can be applied to calculate the hydraulic aperture for closed fractures [43]:

$e=\frac{e_{0}}{1+9 \frac{\bar{\sigma}}{\sigma^{*}}}+\delta \tan \frac{\varphi}{1+9 \frac{\bar{\sigma}}{\sigma^{*}}}$,

where $e_{0}$ and $\sigma^{*}$ are laboratory-derived constants that define the stiffness of closed fractures, and $\varphi$ is called the dilation angle. For open fractures, the hydraulic aperture is calculated as:

$e=e_{0}+\Delta e+\delta \tan \varphi$.

Equations 19 and 20 ensure that fracture aperture remains continuous as fractures transition between closed and open states. A distinction is made between the fracture hydraulic aperture, related to fluid flux, and fracture void aperture, related to fluid storativity, in order to account for complexities that exist in reality which cannot be captured at the scale of the model. Equivalent relationships to Eqs. 19 and 20 are used to calculate the void aperture, $E$, and the constants are allowed to be different.

\subsection{Fracture propagation}

In this work, only purely mode-I fracture propagation was considered. Hydraulic fractures were assumed to propagate in the direction parallel to the maximum principal stress orientation. Fracture propagation was assumed to occur subject to the critical stress intensity factor criterion. The mode-I stress intensity factor, $K_{I}$, at fracture tips was calculated as a function of the opening mode displacement discontinuity [30]:

$K_{I}=0.806\left[\frac{E}{4\left(1-v^{2}\right)}\right]\left(\frac{\pi}{a}\right)^{1 / 2} \Delta e$

where $E$ is Young's modulus, $v$ is Poisson's ratio, $a$ is the length of the fracture tip element, and $\Delta e$ in this case is the mode-I displacement discontinuity of the fracture tip element. In the model, when the value of $K_{I}$ reaches the critical stress intensity factor, $K_{I C}$, the fracture is allowed to propagate, and an additional fracture element is appended to the fracture. Once a new fracture element nucleates, the appropriate matrix-fracture mass transfer term is activated in the mass balance equations (see Eqs. 1 and 2).

\section{Injection into an infinite-conductivity fracture}

The EFM approach has been verified by previous authors for a range of physical scenarios of practical interest, including black oil [21], geothermal [15], unconventional gas [26], and injection-induced seismicity [16, 29]. Here, a numerical example is shown to demonstrate the validity of the present model. Norbeck et al. [27] observed previously that the EFM implementation achieved a suitable degree of accuracy for a problem involving 1-D leakoff from a single fracture that connected an injection and production well. This was a relatively benign scenario because the EFM fracture index (see Eq. 9) was derived assuming linear flow near the fracture.

In this example, injection into a vertical infiniteconductivity fracture in an infinite reservoir was considered. The reservoir response for this problem is a linear flow regime at early times, followed by a transition to radial flow at later times. A closed-form solution exists for the transient pressure response at the well [8]:

$$
\begin{aligned}
p_{D}^{w}\left(t_{D}\right)= & 0.5 \sqrt{\pi t_{D}}\left[\operatorname{erf}\left(\frac{0.134}{\sqrt{t_{D}}}\right)+\operatorname{erf}\left(\frac{0.866}{\sqrt{t_{D}}}\right)\right] \\
& +0.067 \mathrm{Ei}\left(\frac{0.018}{t_{D}}\right)+0.433 \mathrm{Ei}\left(\frac{0.750}{t_{D}}\right) .
\end{aligned}
$$


Table 1 Model parameters for injection into an infinite-conductivity fracture

\begin{tabular}{lll}
\hline Parameter & Value & Unit \\
\hline$x_{f}$ & 50 & $\mathrm{~m}$ \\
$h_{f}$ & 10 & $\mathrm{~m}$ \\
$e$ & 0.01 & $\mathrm{~m}$ \\
$k^{m}$ & $20 \times 10^{-15}$ & $\mathrm{~m}^{2}$ \\
$\phi$ & 0.2 & - \\
$c$ & $8.8 \times 10^{-10}$ & $\mathrm{~Pa}^{-1}$ \\
$\mu$ & 0.001 & $\mathrm{~Pa} \cdot \mathrm{s}$ \\
$q^{w}$ & 0.01 & $\mathrm{~m}^{3} \cdot \mathrm{s}^{-1}$ \\
$p_{0}$ & 40 & $\mathrm{MPa}$ \\
\hline
\end{tabular}

Here, the dimensionless pressure variable, $p_{D}^{w}$, is defined as:

$p_{D}^{w}=\frac{2 \pi k^{m} h_{f}}{q^{w} \mu}\left(p^{w}-p_{0}\right)$,

and the dimensionless time variable, $t_{D}$, is defined as:

$t_{D}=\frac{k^{m}}{\mu \phi c x_{f}^{2}} t$,

where $h_{f}$ is the fracture height (which is equal to the reservoir thickness), $q^{w}$ is the volumetric injection rate, $p^{w}$ is the wellbore pressure, $p_{0}$ is the initial reservoir pressure, $x_{f}$ is the fracture half-length, and $t$ is the time since the start of injection.

\subsection{Problem description}

The model parameters used in the simulations are given in Table 1. In the model, fluid was injected at a constant rate for a period of $48 \mathrm{~h}$. Wellbore storage effects were neglected. Geomechanical effects were not considered, so the properties of the fracture remained constant throughout the simulations (i.e., the length and permeability of the fracture were fixed).

The EFM, DFM, and 1-D leakoff approximation models were compared. In each case, the same fracture discretization was used. For the EFM nonconforming matrix discretization, a structured Cartesian mesh comprised of 90,601 control volumes was used. For the DFM conforming matrix discretization, a triangular mesh comprised of 80,876 control volumes was used.

\subsection{Numerical results}

The wellbore pressure response curves observed in the three simulations are compared against Eq. 22 on a log-log plot in Fig. 3. The time derivative of the EFM pressure response was evaluated numerically, and is also plotted in the same figure. The $1 / 2$ slope of both the pressure and pressure derivative curves at early times is indicative of the linear flow regime that developed when flow was dominated by the presence of the fracture [10]. Each of the three numerical models captured this behavior accurately. There are slight

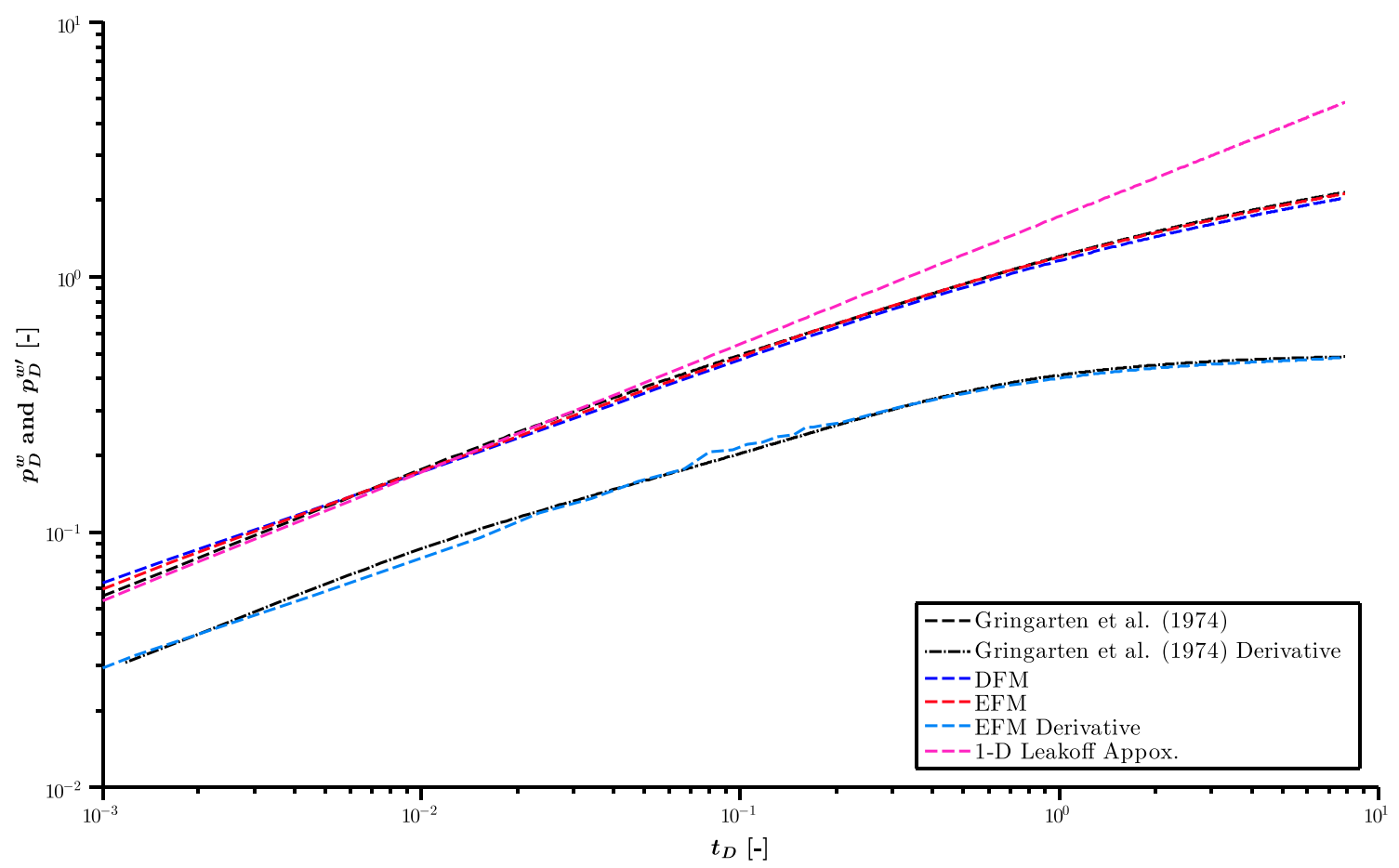

Fig. 3 Log-log plot of pressure and pressure derivative in dimensionless space for the infinite-conductivity fracture injection problem illustrated in Section 4 
discrepancies observed for the EFM and DFM models at very early time $\left(t_{D}<4 \times 10^{-3}\right)$, but the magnitude of the errors are small and appear exaggerated in the figure due to the log scale. The 1-D leakoff approximation performed extremely well in the linear flow regime.

At a time of $t_{D} \approx 4 \times 10^{-2}$, the pressure response calculated using Eq. 22 begins to diverge from the linear flow regime and transitions towards a radial flow regime. From Fig. 3, it is observed that both the EFM and DFM models were able to capture the transition from linear to radial flow accurately. As expected, the 1-D leakoff approximation was not valid for simulation times that extended well beyond the linear flow regime.

In addition to demonstrating the accuracy of the three numerical models, this example also revealed a very interesting characteristic of the EFM approach. While the fracture index embodies an implicit assumption that flow is purely linear in the vicinity of the fracture, in this example the global pressure response of late-time radial flow was captured accurately. In this light, it is evident that the concept of EFM may be applied in scenarios that involve more complex flow regimes, for example, in naturally fractured reservoirs in which nearby fractures may affect each other.

\section{Shear stimulation}

In geothermal settings, it is commonly assumed that hydraulic stimulation occurs due to shear stimulation, a process in which fluid injection triggers slip and permability enhancement on natural fractures. Shear stimulation involves injecting fluid at a pressure less than the magnitude of the minimum principal stress into a network of prexisting natural fractures in order to reduce the effective normal stress acting on the individual fracture planes and thereby reducing their resistance to shear failure. The premise is that some types of rock may be conducive to a self-propping behavior upon shear failure that can lead to a permanent enhancement of permeability [20]. In addition, shear failure of natural fractures is responsible for causing microseismic events that are observed commonly during hydraulic fracture treatments, and so principles related to shear stimulation can be applied to interpret the microseismic activity generated during hydraulic fracturing [40].

During shear stimulation treatments, the emergent behavior of an altered reservoir transmissivity is a very nonlinear process involving a tight coupling between fluid flow, mechanical deformation of fractures, shear failure of fractures, and a constitutive relationship describing fracture permeability evolution. As fractures deform, stresses are transferred elastically throughout the domain, and it can be extremely important to solve the full geomechanics problem in order to resolve these effects. McClure and Horne
[22] demonstrated that stress transfer effects can influence the rate at which shear stimulation propagates along individual fractures significantly, giving rise to a mechanism they called crack-like shear stimulation. Localized shear stress concentrations develop ahead of the zone that has previously experienced slip and can cause slip to occur before the pressure front reaches that location, further enhancing permeability and promoting flow. In this manner, shear stimulation is able to propagate at a rate related to the enhanced fracture transmissivity, rather than the initial fracture transmissivity. Considering this mechanism, models that employ simplified geomechanical models based purely on pressure diffusion, such as those presented by Moinfar et al. [26] or Karvounis et al. [16], may not be sufficient to predict reservoir response realistically in many injection and production scenarios of practical interest. The numerical simulation results presented in this section demonstrate that the EFM approach can be applied to solve coupled fluid flow and geomechanics problems with nonlinear fracture permeability evolution.

\subsection{Problem description}

In this numerical example, shear stimulation of a relatively complex network of preexisting fractures was considered. The domain was essentially 2-D (i.e., all fractures were vertical and had fixed heights equal to the reservoir thickness). The stress regime was strike-slip. A $150 \mathrm{~m}$ section of a horizontal well-penetrated several natural fractures. Fluid was injected at a constant pressure such that the reservoir fluid pressure never exceeded the minimum principal stress, ensuring that tensile fractures would not propagate. Therefore, changes in injectivity were purely due to permeability changes caused by the shear stimulation effect. The fracture network and well geometry are illustrated in Fig. 4, and the model parameters are listed in Table 2.

Fluid was injected for a period of 7 days at a constant pressure of 12.4 MPa. The magnitude of the minimum principal stress was 14.5 MPa. The metrics for comparison are injection rate as a function of time, cumulative mass transfer between the matrix and fracture domains as a function of time, and the spatial distribution of shear displacement throughout the fracture network at the end of the stimulation treatment. The DFM results should be considered the "most true" solutions because they employed the standard conforming discretization strategy that is commonly used in reservoir simulation practice.

A series of four test cases were modeled. In cases 1-3, the results of the EFM, DFM, 1-D leakoff approximation model, and zero leakoff approximation model were compared for a range of matrix permeabilities. In case 4 , a discretization refinement study for the EFM was performed. The same fracture network discretization, with a total of 
Fig. 4 Plan view of the fracture network geometry for the shear stimulation example illustrated in Section 5. The black line is the horizontal wellbore and the blue lines are the natural fractures

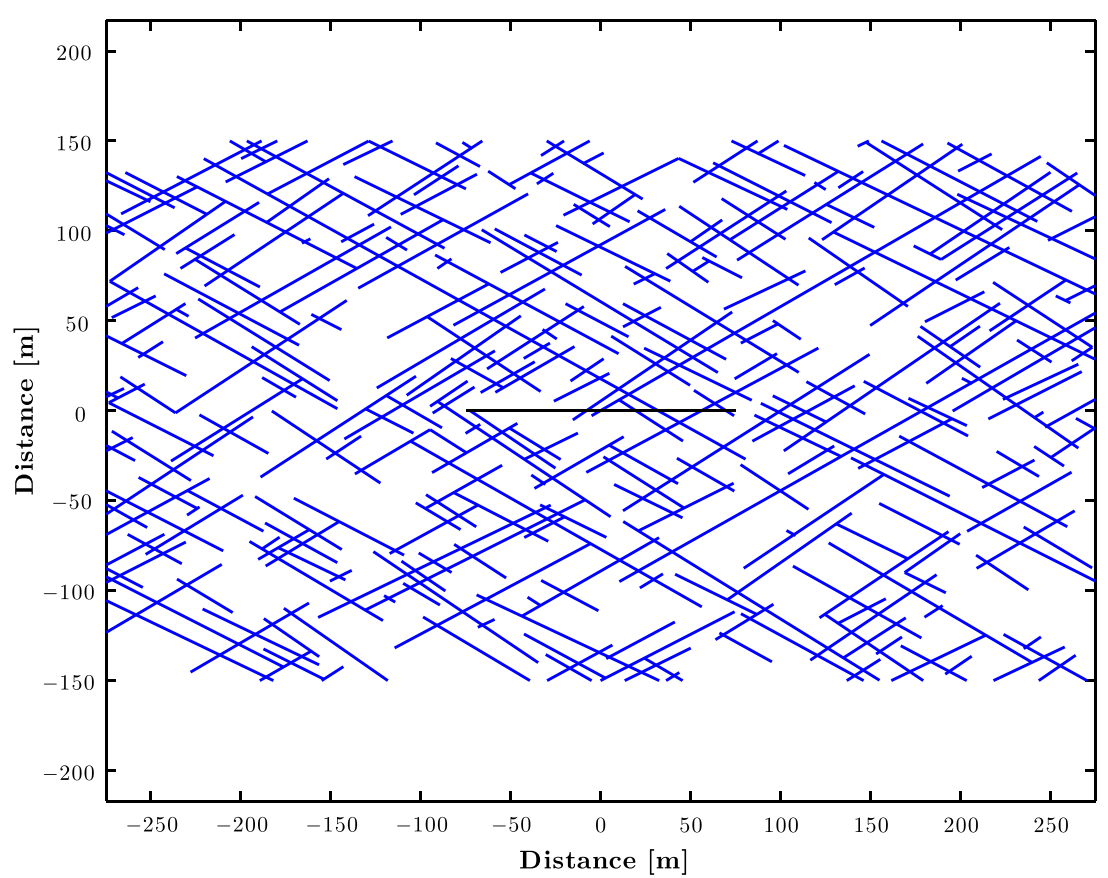

16,547 fracture control volumes, was used in each of the simulations. In cases 1-3, the EFM employed a nonconforming structured Cartesian mesh with 251,001 control volumes for the matrix discretization. The DFM employed a conforming triangular mesh with 351,569 control volumes

Table 2 Model parameters for study on shear stimulation in a naturally fractured reservoir

\begin{tabular}{lll}
\hline Parameter & Value & Unit \\
\hline$h_{f}$ & 100 & $\mathrm{~m}$ \\
$e_{0}$ & $5 \times 10^{-5}$ & $\mathrm{~m}$ \\
$E_{0}$ & $5 \times 10^{-3}$ & $\mathrm{~m}$ \\
$\sigma_{e}^{*}$ & 90 & $\mathrm{MPa}$ \\
$\sigma_{E}^{*}$ & 90 & $\mathrm{MPa}$ \\
$\varphi_{e}$ & 5 & $\mathrm{deg}$. \\
$\varphi_{E}$ & 0 & $\mathrm{deg}$. \\
$f$ & 0.6 & - \\
$\eta$ & 3 & $\mathrm{MPa} \cdot \mathrm{m}^{-1} \cdot \mathrm{s}$ \\
$k^{m}$ & $10^{-19}$ to $10^{-17}$ & $\mathrm{~m}$ \\
$\phi_{0}$ & 0.03 & - \\
$c_{\phi}$ & $1.9 \times 10^{-9}$ & $\mathrm{~Pa}^{-1}$ \\
$\rho_{0}$ & 1000 & $\mathrm{~kg} \cdot \mathrm{m}^{-3}$ \\
$c_{w}$ & $4.6 \times 10^{-10}$ & $\mathrm{~Pa}^{-1}$ \\
$\mu$ & 0.001 & $\mathrm{~Pa} \cdot \mathrm{s}$ \\
$p_{0}$ & 8.7 & $\mathrm{MPa}$ \\
$\sigma_{x x}^{R}$ & 23.8 & $\mathrm{MPa}$ \\
$\sigma_{y y}^{R}$ & 14.5 & $\mathrm{MPa}$ \\
$\sigma_{x y}^{R}$ & 0 & $\mathrm{MPa}$ \\
\hline & &
\end{tabular}

for the matrix discretization. The 1-D and zero leakoff approximation models did not require discretization of the matrix rock volume. In case 4, the number of EFM matrix control volumes was varied from 121 to 251,001 to test for the property of convergence upon refinement and to evaluate the EFM's ability to obtain suitable degrees of accuracy with coarser grids.

\subsection{Numerical results}

The flow behavior in the reservoir was affected strongly by the geometry, connectivity, and hydraulic properties of the natural fracture network. The EFM pressure distribution in the matrix rock at the end of the simulation, shown in Fig. 5, shows that fluid leakoff was limited to regions close to the fractures. Flow evidently occurred preferentially along a relatively extensive natural fracture in the upper-right corner of the domain.

The main comparison metric for these numerical experiments is injection rate as a function of time. The injection profiles for each case are shown in Fig. 6. Case 1 represents the base case simulation. The matrix permeability was $k^{m}=1 \times 10^{-18} \mathrm{~m}^{2}$. The injection rate during the stimulation for case 1 is shown in Fig. 6a. Because the boundary condition on the injection well was constrained at a constant pressure, sudden increases in the slope of the injection rate corresponded to shear stimulation events. As the critical pressure required to cause shear failure was reached at one particular location, the resulting elastic stress transfer tended to cause cascading failure in nearby fractures, 
which is the reason why several distinct stimulation events are observed in Fig. 6a.

For case 1, the EFM and DFM results are indistinguishable, suggesting that EFM was able to obtain a high degree of accuracy in the global flow behavior. To further verify the accuracy of the EFM results, the total amount of fluid that leaked off from the fractures into the matrix domain was compared to the DFM simulation and is shown in Fig. 7. The cumulative mass exchange for both models was normalized by the total mass of fluid injected in the DFM simulation. It is clear that by treating the mass exchange as source terms via the EFM framework, the matrix-fracture mass exchange behavior was captured accurately for a complex fracture geometry. These results demonstrate that the EFM approach can indeed perform well for problems involving coupled flow, geomechanics, and nonlinear permeability evolution in highly fractured reservoirs.

For case 1, the 1-D leakoff model performed well in terms of capturing the timing and magnitude of the major shear stimulation events and the late time injection rate. The zero leakoff model predicted that shear stimulation events occurred relatively early, which is physically intuitive because pressures everywhere in the fracture network were higher without the leakoff effect. It is interesting to note that each of the four models showed the same general trends in reservoir response, and the effect of fluid leakoff evidently impacted only the onset of each individual stimulation event.

Figure 6b, c show the injection rate histories for cases 2 and 3, which correspond to matrix permeabilities of $k^{m}=$ $0.1 \times 10^{-18} \mathrm{~m}^{2}$ and $k^{m}=10 \times 10^{-18} \mathrm{~m}^{2}$, respectively. For each of these cases, the EFM and DFM injection profiles were nearly identical. For case 2 , which had a relatively low matrix permeability, the range of predictions for the four models narrowed significantly. The full matrix discretization approaches provided only modest improvements over the 1-D leakoff approximation. For case 3, which had a relatively high matrix permeability, the results were again consistent with intuition. The range in model predictions was largest for this case because fluid leakoff effects were more pronounced than in any of the previous cases. At later times, the 1-D leakoff approximation predicted a significantly higher injection rate than the EFM and DFM simulations. This can be attributed to the fact that for nearby fractures, the distance of investigation of the pressure transient was large enough that they began to affect each other. The 1-D leakoff model is fundamentally unable to resolve this behavior.

For the EFM discretization refinement study, seven levels of matrix refinement were tested. Both the zero leakoff and 1-D leakoff approximation results are also shown for reference. In all cases, the same fracture discretization was used. The matrix permeability was the same as case $1\left(k^{m}=\right.$ $1 \times 10^{-18} \mathrm{~m}^{2}$ ). The injection rate history is shown in Fig. $6 \mathrm{~d}$, and the error in the fracture shear displacement at the end of the simulations is shown in Fig. 8. The results indicate that the EFM approach was convergent upon grid refinement. More interestingly, a high degree of accuracy was still obtained even after a significant reduction in the total number of degrees of freedom. In this example, reducing the number of control volumes from roughly 250,000 down to 90,000 resulted in a negligible loss of accuracy. Further reducing the number of control volumes to about 40,000 still gave acceptable results in terms of predicting a similar injection rate curve and maintaining shear displacement errors less than $5 \%$. Note that for a given fracture discretization,
Fig. 5 Matrix pressure distribution at the end of the stimulation treatment (EFM case 1 of the shear stimulation example in Section 5). The color bar scale ranges from 8.7 to 12.4 MPa

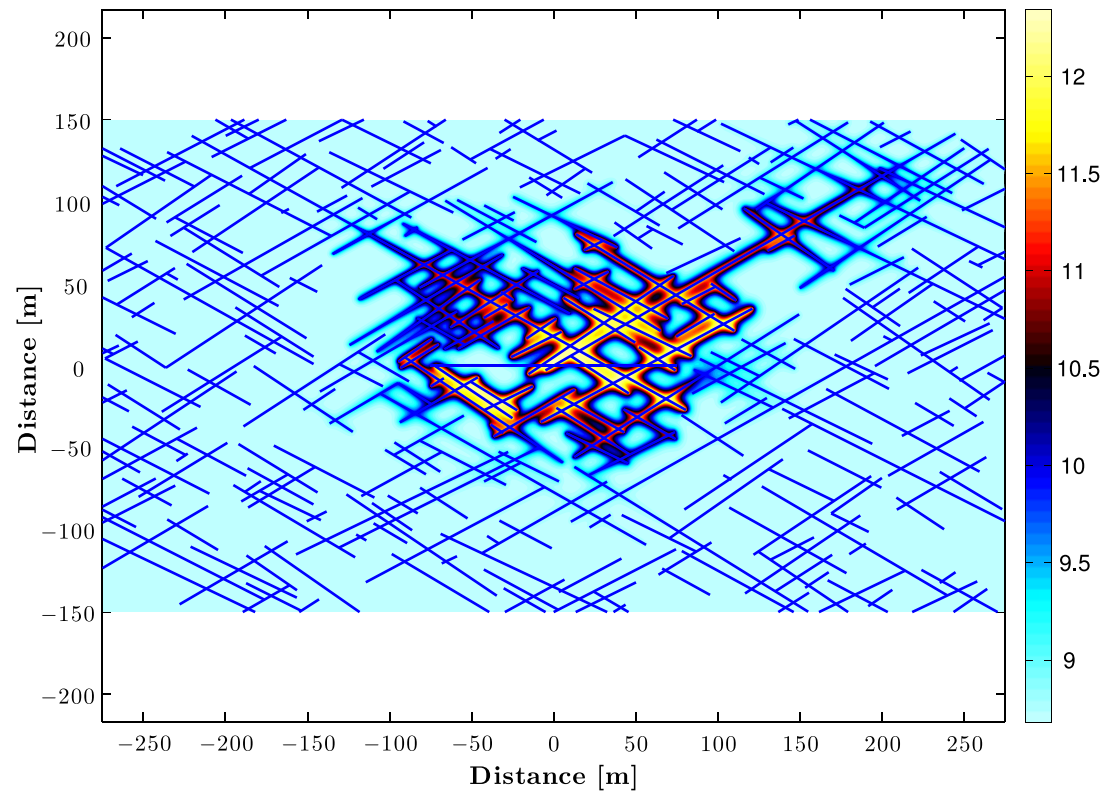


it is difficult to arbitrarily reduce the number of degrees of freedom for conforming discretization techniques such as DFM.

\section{Hydraulic fracture propagation}

Hydraulic fracturing and horizontal drilling are two of the key technological advancements that have allowed for the economic development of unconventional shale gas and shale oil resources. Hydraulic fracturing may also prove to be key for the future development of engineered geothermal systems [36]. In general, the goal of a hydraulic fracture treatment is to expose the wellbore to a larger reservoir surface area in order to enhance recovery rates and ultimate recovery. The process involves injecting fluid at a pressure greater than the magnitude of the minimum principal stress so that new tensile fractures nucleate and propagate through the reservoir. In many applications, the desired effect of a hydraulic fracture treatment is to create a set of large, planar vertical fractures that extend laterally away from the horizontal wellbore.

One technique that is commonly applied for the design of hydraulic fracture treatments was due to Geertsma and de Klerk [6], who introduced what is referred to as the KGD fracture model. For vertical KGD fractures, plane strain conditions are assumed in the vertical direction such that each horizontal cross-section of the fracture has the same geometry. Gidley et al. [7] provided a closed-form solution that describes fracture half-length as a function of time for
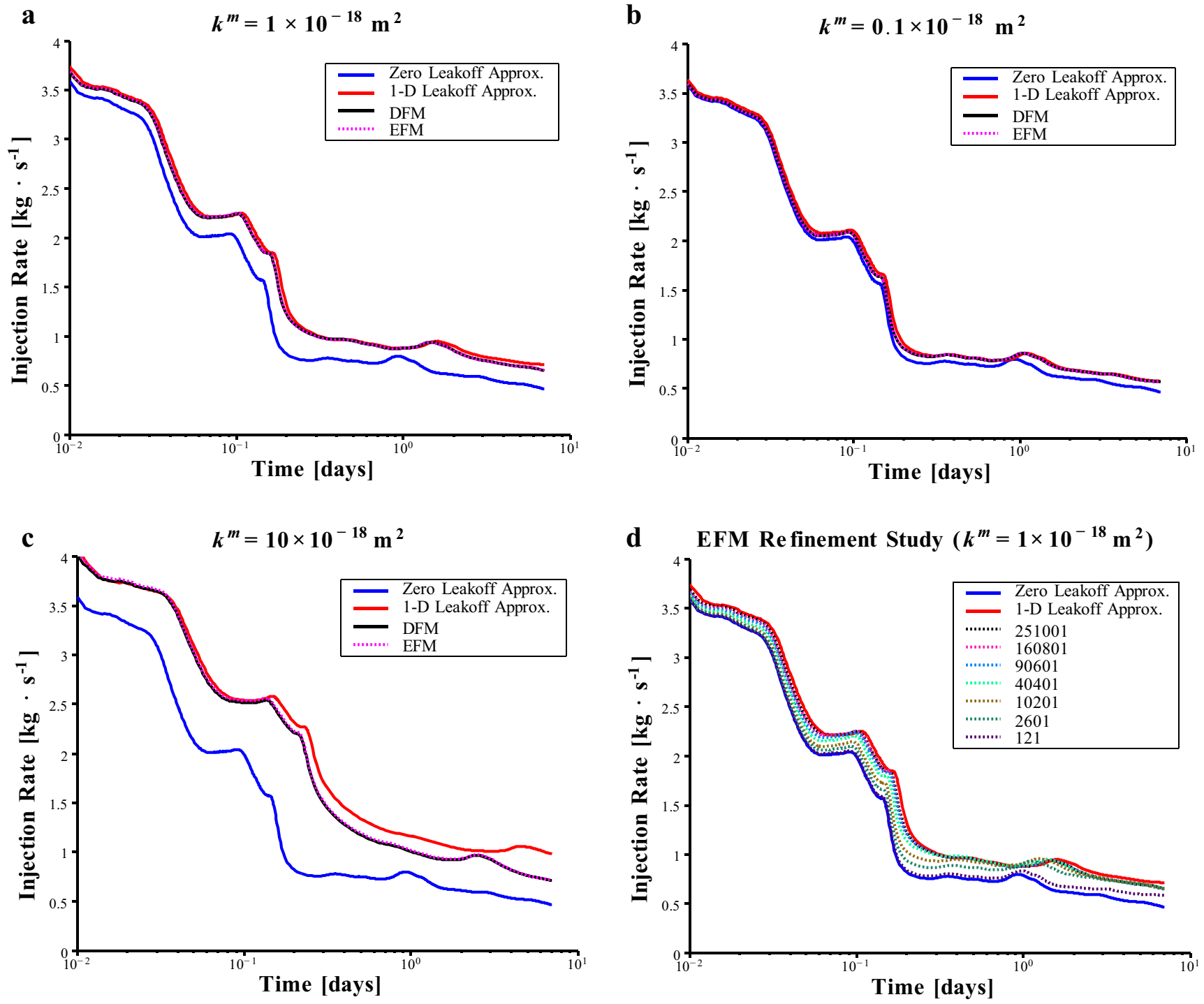

Fig. 6 Injection rate over the duration of the stimulation treatment for cases 1-4 of the shear stimulation example in Section 5 


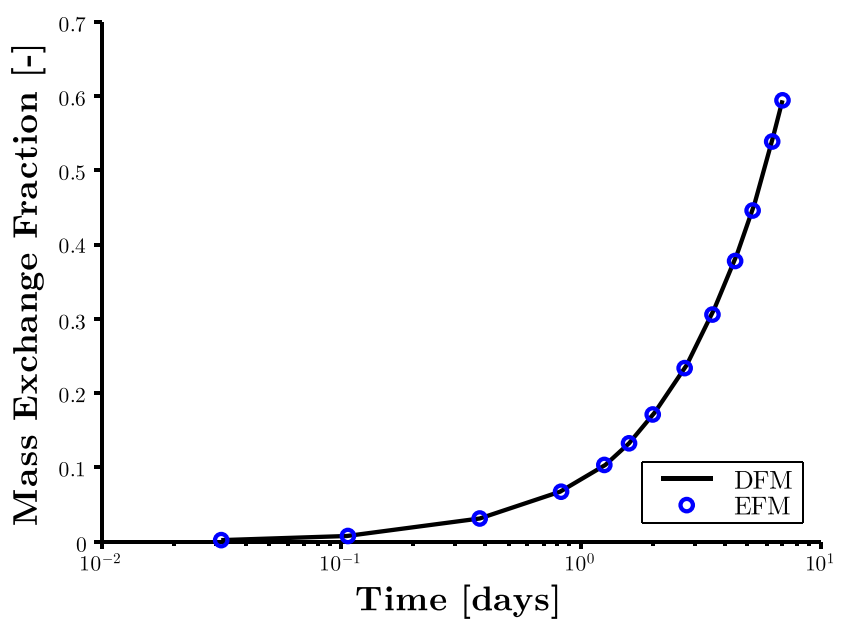

Fig. 7 Cumulative mass transfer between matrix and fracture domains normalized by the total mass of water injected over the entire stimulation treatment for case 1 of the shear stimulation example in Section 5

constant injection rate and assuming no leakoff of fluid into the formation:

$x_{f}=0.679\left[\frac{G i^{3}}{(1-v) \mu h_{f}^{3}}\right]^{1 / 6} t^{2 / 3}$,

where $i$ is half of the total volumetric injection rate (i.e., the flow rate entering one of the fracture wings), and $G$ is shear modulus. For the case where leakoff is considered, Valko and Economides [39] performed a material balance

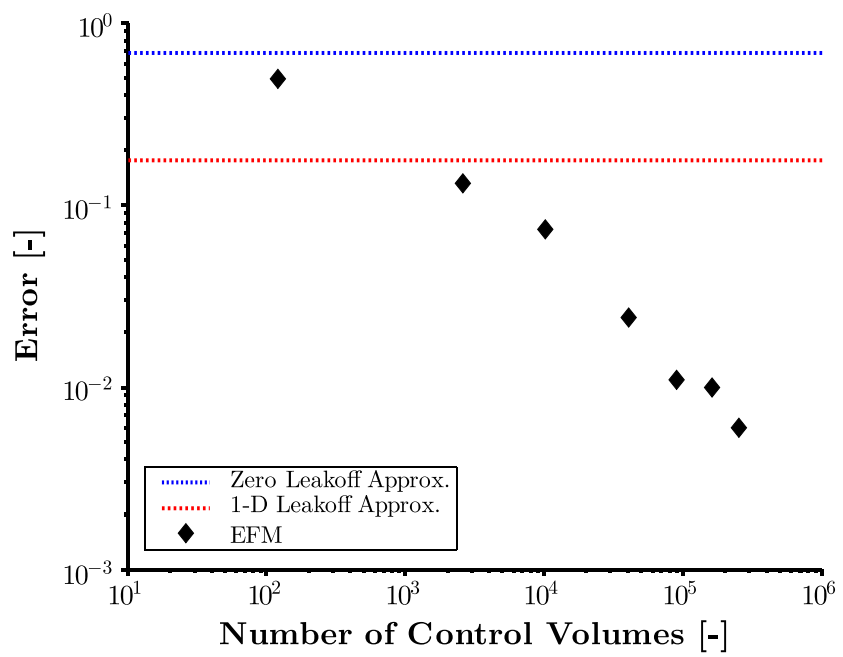

Fig. 8 The error in cumulative shear displacement at the end of the stimulation treatment for various levels of EFM grid refinement (shear stimulation example in Section 5). The blue and red lines represent the error for the zero leakoff and 1-D leakoff approximations, respectively
Table 3 Model parameters for mode-I hydraulic fracture propagation problem

\begin{tabular}{lll}
\hline Parameter & Value & Unit \\
\hline$i$ & 0.05 & $\mathrm{~m}^{3} \cdot \mathrm{s}^{-1}$ \\
$h_{f}$ & 100 & $\mathrm{~m}$ \\
$k^{m}$ & $0.1 \times 10^{-15}$ & $\mathrm{~m}^{2}$ \\
$\phi_{0}$ & 0.2 & - \\
$c$ & $8.8 \times 10^{-10}$ & $\mathrm{~Pa}-1$ \\
$\mu$ & 0.001 & $\mathrm{~Pa} \cdot \mathrm{s}$ \\
$G$ & 15 & $\mathrm{GPa}$ \\
$v$ & 0.25 & - \\
$K_{I C}$ & 1 & $\mathrm{MPa} \cdot \mathrm{m}^{1 / 2}$ \\
$p_{0}$ & 40 & $\mathrm{MPa}$ \\
$\sigma_{3}$ & 45 & $\mathrm{MPa}$ \\
$\Delta p$ & 5.2 & $\mathrm{MPa}$ \\
$C_{L}$ & $7.8 \times 10^{-6}$ & $\mathrm{~m} \cdot \mathrm{s}^{-1 / 2}$ \\
\hline
\end{tabular}

that yielded a nonlinear function for fracture length (given here, neglecting spurt-loss):

$x_{f}=\frac{\bar{e} i}{4 \pi C_{L}^{2} h_{f}}\left[\exp \left(\beta^{2}\right) \operatorname{erfc}(\beta)+\frac{2 \beta}{\sqrt{\pi}}-1\right]$,

where $\bar{e}$ is the average aperture of the hydraulic fracture and $C_{L}$ is the leakoff coefficient. The time variable is included in the parameter, $\beta$ :

$\beta=\frac{2 C_{L} \sqrt{\pi t}}{\bar{e}}$.

The nonlinearity arises because the average fracture aperture at any time depends on the length of the fracture.

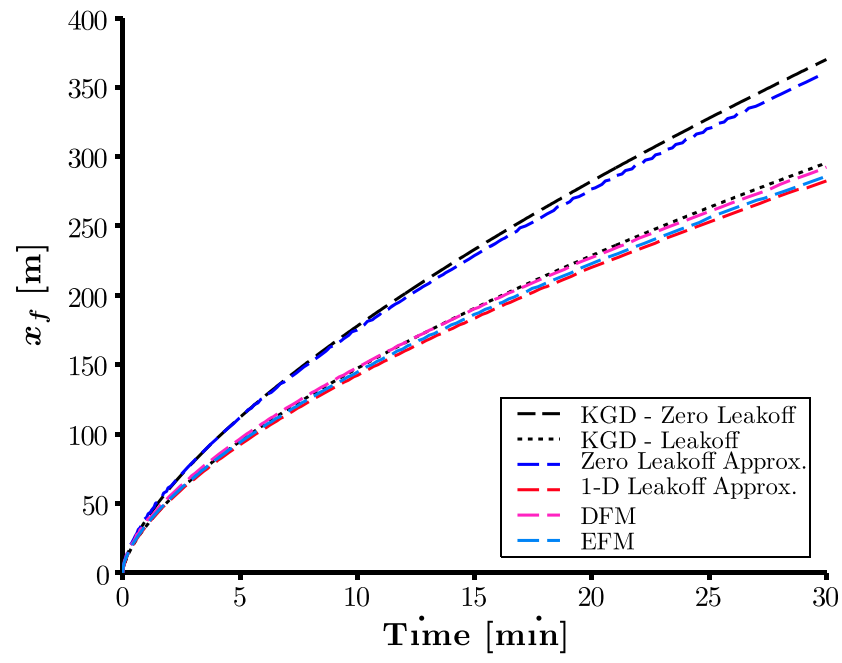

Fig. 9 Hydraulic fracture half-length as a function of injection time for the fracture propagation example illustrated in Section 6. Comparison of the four different models to the semianalytical solutions (see Eqs. 25 and 26) 
Table 4 Fracture half-length at end of injection for the model comparison study

\begin{tabular}{lllllll}
\hline & Eq. 25 & Zero Leakoff Model & Eq. 26 & 1-D Leakoff Model & DFM & EFM \\
\hline$x_{f}(\mathrm{~m})$ & 370.0 & 360.0 & 295.5 & 282.5 & 292.5 & 285.0 \\
Difference (\%) & N/A & -2.7 & N/A & -4.4 & -1.0 & -3.6 \\
\hline
\end{tabular}

Gidley et al. [7] suggest that the fracture aperture at the wellbore, $e_{w}$, is:

$e_{w}=2.27\left[\frac{\mu x_{f}(1-v) i}{G h_{f}}\right]^{1 / 4}$.

For the KGD fracture geometry, the average value of the fracture aperture is:

$\bar{e}=\frac{\pi}{4} e_{w}$.

If the leakoff coefficient is known, Eqs. 27-29 can be substituted into Eq. 26, so that Eq. 26 becomes only a function of time and fracture length.

In this study, leakoff from the fracture was considered to be slightly compressible flow driven purely by diffusion into the matrix rock. Under this assumption, the leakoff coefficient, $C_{L}$, is [5]:

$C_{L}=\left[\frac{k^{m} \phi_{0} c}{\pi \mu}\right] \Delta p$,

where $\Delta p=p^{f}-p_{0}$ is the pressure drop driving leakoff.

\subsection{Problem description}

In this numerical example, the models' ability to simulate pure mode-I fracture propagation was examined. Propagation of a single two-wing vertical fracture was considered. The domain was assumed to be homogeneous in terms of fluid flow and mechanical properties, and no preexisting natural fractures were present. Plane strain conditions in the vertical direction were assumed, so the numerical solutions are compared with KGD semianalytical solutions [7, 39]. Three sets of simulations were performed. In the first case, the matrix rock was assumed to be completely impermeable, such that no leakoff occurred. The zero leakoff model is compared to Eq. 25. In the second case, the matrix rock was assigned a permeability value of $k^{m}=0.1 \times 10^{-15} \mathrm{~m}^{2}$ in order to investigate the effects of leakoff. The 1-D leakoff

Table 5 Reduction in fracture half-length due to the leakoff effect for the model comparison study

\begin{tabular}{ll}
\hline Model & Reduction in $x_{f}(\%)$ \\
\hline Semianalytical KGD & 20.1 \\
1-D Leakoff Model & 21.5 \\
DFM & 18.8 \\
EFM & 20.8 \\
\hline
\end{tabular}

model, EFM, and DFM are compared to Eq. 26. In the third case, a discretization refinement study for the EFM was performed under the permeable rock scenario. The metric used for comparison is the hydraulic fracture length as a function of time.

The model parameters for this problem are given in Table 3. In the model, fluid was injected at a constant rate of $0.05 \mathrm{~m}^{3} \cdot \mathrm{s}^{-1}$ for $30 \mathrm{~min}$. The hydraulic fracture had a fixed height of $100 \mathrm{~m}$. The magnitude of the least principal stress was $5 \mathrm{MPa}$ above the initial reservoir pressure, so the relatively high pressure in the fracture necessary to drive propagation encouraged leakoff to occur for the case of permeable matrix rock. To determine the leakoff coefficient used to calculate the semianalytical solution to the problem (see Eq. 30), the pressure drop was assumed to be $\Delta p=5.2 \mathrm{MPa}$. This value was based on the average pressure in the fracture observed during the numerical simulations. The same fracture discretization was used for the DFM and EFM simulations. The DFM and EFM matrix discretizations had 102,012 and 160,801 control volumes, respectively.

In general, the numerical models are not expected to achieve an exact match with the semianalytical solutions because there are some significant differences in the respective underlying assumptions. For example, the semianalytical solutions are based purely on volume balances, whereas the numerical solutions allow fracture propagation to occur

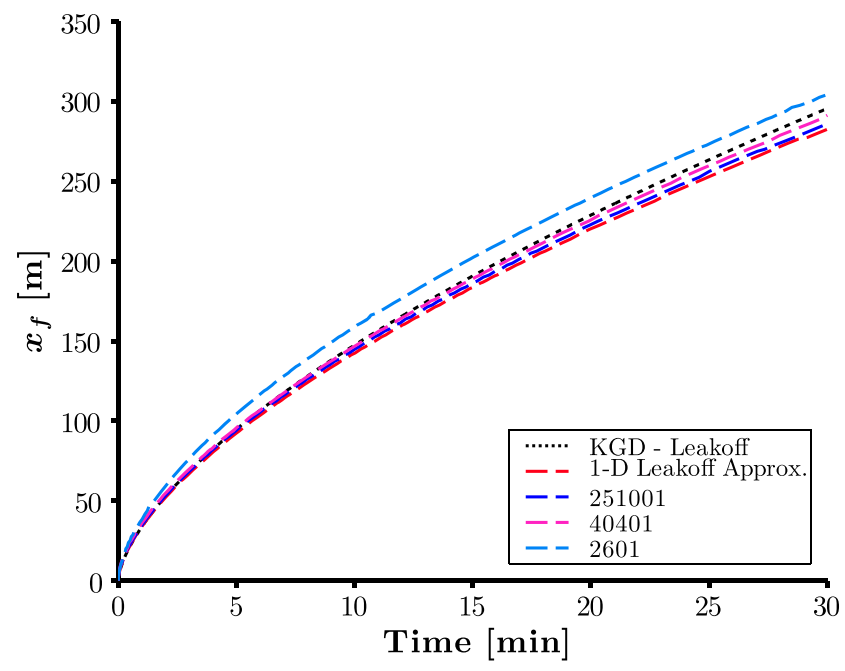

Fig. 10 EFM grid refinement study for fracture propagation example illustrated in Section 6. The solutions are convergent upon grid refinement and appear to be converging towards the 1-D leakoff approximation. The results from the simulation with 90,601 control volumes are not shown here for the sake of clarity 
Table 6 Fracture half-length at end of injection for the EFM discretization refinement study

\begin{tabular}{lllllll}
\hline & Eq. 26 & 1-D Leakoff Model & 251001 & 90601 & 40401 & 2601 \\
\hline$x_{f}(\mathrm{~m})$ & 295.5 & 282.5 & 285.0 & 288.7 & 291.3 & 303.8 \\
Difference $(\%)$ & N/A & -4.4 & -3.6 & -2.3 & -1.4 & 2.8 \\
\hline
\end{tabular}

subject to a criterion based on the mode-I stress intensity factor.

\subsection{Numerical results}

The comparison between the numerical and semianalytical models of the temporal evolution of fracture length is illustrated in Fig. 9. The numerical models captured the fracture growth behavior very well over the duration of injection. The numerical solutions matched the semianalytical solutions extremely accurately at early times during the period of rapid growth of the fracture. The numerical models tended to underestimate the fracture length slighly at later times. A summary of the fracture half-length at the time fluid injection stopped is given in Table 4. For the case of no leakoff, the zero leakoff approximation model underestimated the KGD solution by $2.7 \%$. For the cases where leakoff occurred, the difference between the numerical and semianalytical models ranged from -1.0 to $-4.4 \%$.

The effect of fluid leakoff during the hydraulic fracture simulations was that some of the fluid was lost into the formation and was no longer useful for creating new fracture volume. A summary of the reduction in fracture length due to leakoff is provided in Table 5. In these calculations, the KGD solutions were compared to each other, and the DFM, EFM, and 1-D leakoff model were each compared to the zero leakoff model. The numerical models predicted a reduction in fracture length ranging from 18.8 to $21.5 \%$, which compares favorably with the semianalytical solution of $20.1 \%$.

The results of the EFM matrix grid refinement study are illustrated in Fig. 10 and summarized in Tables 6 and 7. For coarse levels of grid refinement, the amount of fluid leakoff tended to be underestimated resulting in longer

Table 7 Reduction in fracture half-length due to the leakoff effect for the EFM discretization refinement study

\begin{tabular}{ll}
\hline Model & Reduction in $x_{f}(\%)$ \\
\hline Semianalytical KGD & 20.1 \\
1-D Leakoff Model & 21.5 \\
251001 & 20.8 \\
90601 & 19.8 \\
40401 & 19.1 \\
2601 & 15.6 \\
\hline
\end{tabular}

hydraulic fractures. The solutions were convergent upon grid refinement, which is an attractive numerical property of EFM. Interestingly, the solutions converged toward the 1-D leakoff approximation solution. Because the duration of injection was very short and the matrix permeability was relatively low, the assumption of 1-D flow away from the newly forming fracture was quite reasonable. In this light, the 1-D leakoff approximation model could be considered the "most true" numerical solution, and it is encouraging that the EFM solution approached the 1-D leakoff model.

Quantitatively, the models performed extremely well. When compared to the semianalytical KGD solutions, the magnitude of the mismatch of the numerical solutions were within reason for practical purposes. It is worth noting that the literature provides several alternatives to Eqs. 25 and 26 that predict differences in fracture half-length on the order of $10 \%$ [39], and the present numerical solutions fall within that range. These results indicate that the EFM framework can be applied successfully to scenarios in which fractures are propagating and fracture systems are growing over time.

\section{Discussion and concluding remarks}

In this work, we developed a novel numerical modeling framework based on the embedded fracture modeling (EFM) approach as an effective technique to model reservoir stimulation processes such as hydraulic fracturing and shear stimulation. The EFM approach was implemented in a reservoir model that couples fluid flow, mechanical deformation, and rock failure processes. In order to verify the accuracy of the embedded fracture model, the present model was compared to a more traditional discrete fracture model (DFM) in three separate numerical examples. In each example, the EFM performed rermarkably well and yielded results that matched the DFM to within an acceptable margin of error.

In this paper, we demonstrated that the EFM is extremely well-suited for fracture propagation problems. In the EFM framework, newly formed fracture control volumes can be integrated into the numerical model with relative ease. The ability to discretize the fracture and matrix rock domains separately ensures that fractures are able to propagate without the numerical constraints associated with traditional approaches that employ conforming meshes. We showed 
that it is possible to coarsen the matrix discretization and still obtain a reasonable degree of accuracy using the EFM approach. Once a fracture discretization has been defined for a DFM, however, it is very difficult to arbitrarily coarsen the matrix discretization in the same fashion that is possible with EFM. This has important implications when moving towards increased problem complexity, for instance, when considering interaction between propagating fractures and natural fractures, branching and curving fractures, or three dimensions. Issues associated with numerical discretization in these complex scenarios can set practical limitations on the utility of reservoir modeling, and are largely overcome in the EFM framework.

Two approximate models were also developed and compared to both EFM and DFM. These models are referred to as the one-dimensional leakoff and zero leakoff approximation models, and were observed to provide useful constraints on reservoir stimulation behavior at significantly reduced computational effort. For very low matrix permeabilities, all models were observed to provide similar results. As matrix permeability increased, the two approximate models diverged from the EFM and DFM models. Further investigation must be performed in order to better classify the range of geologic and operational parameters over which each of the models retain a high level of accuracy.

It has become clear that geomechanics can play an important role in many different facets of reservoir engineering practice. For example, mechanisms that enable permeability creation during hydraulic fracturing are controlled largely by mechanical effects. Shear slip events commonly observed during microseismic monitoring operations of fluid injection treatments help reservoir engineers define the stimulated region. The reservoir model described in this paper can be applied in practical settings to help design and optimize reservoir management strategies or in research settings to better understand fundamental reservoir processes.

Acknowledgments We thank the industrial affiliates of the Stanford Center for Induced and Triggered Seismicity for partial financial support of this work. The financial support of the Cockrell School of Engineering at The University of Texas at Austin is also gratefully acknowledged. The numerical simulations were performed at the Stanford Center for Computational Earth and Environmental Science (CEES) using their high performance computing (HPC) resources.

Open Access This article is distributed under the terms of the Creative Commons Attribution 4.0 International License (http:// creativecommons.org/licenses/by/4.0/), which permits unrestricted use, distribution, and reproduction in any medium, provided you give appropriate credit to the original author(s) and the source, provide a link to the Creative Commons license, and indicate if changes were made.

\section{References}

1. Aziz, K., Settari, A.: Petroleum Reservoir Simulation. Khalid Aziz and Anonin Settari (1979)

2. Bird, R.B., Stewart, W.E., Lightfoot, E.N.: Transport Phenomena. 2nd edn. Wiley (2006)

3. Crouch, S.L., Starfield, A.M.: Boundary Element Methods in Solid Mechanics. Allen and Unwin, London (1983)

4. Ding, D.Y., Wu, Y.S., Jeannin, L.: Efficient simulation of hydraulic fractured wells in unconventional reservoirs. J. Pet. Sci. Eng. 122, 631-642 (2014)

5. Economides, M.J., Nolte, K.G.: Reservoir Stimulation. 3rd edn. Wiley (2000)

6. Geertsma, J., de Klerk, F.: A rapid method of predicting width and extent of hydraulically induced fractures. SPE J. 21(12), 15711581 (1969)

7. Gidley, J.L., Holditch, S.A., Nierode, D.E., Veatch Jr. R.W.: Recent advances in hydraulic fracturing. SPE Monogr. Ser. 12 (1990)

8. Gringarten, A.C., Ramey Jr. H.J., Raghavan, R.: Unsteadystate pressure distributions created by a well with a single infinite-conductivity vertical fracture. SPE J. 14(4), 347-360 (1974)

9. Hajibeygi, H., Karvounis, D., Jenny, P.: A hierarchical fracture model for the iterative multiscale finite volume method. J. Comp. Phys. 230(24), 8729-8743 (2011)

10. Horne, R.N. Modern Well Test Analysis: A Computer-Aided Approach, 2nd edn. Petroway Inc., Palo Alto (1995)

11. Howard, G., Fast, C.R.: Optimum fluid characteristics for fracture extension. Drill. Prod. Pract. 24, 261-270 (1957)

12. Hunsweck, M.J., Shen, Y., Lew, A.J.: A finite element approach to the simulation of hydraulic fractures with lag. Int. J. Numer. Anal. Meth. Geomech. 37, 993-1015 (2013)

13. Jaeger, J.C., Cook, N.G.W., Zimmerman, R. Fundamentals of Rock Mechanics, 4th edn. Blackwell Publishing Ltd., Oxford (2007)

14. Karimi-Fard, M., Durlofsky, L., Aziz, K.: An efficient discretefracture model applicable for general purpose reservoir simulators. SPE J. 9(2), 249-262 (2004)

15. Karvounis, D.: Simulations of Enhanced Geothermal Systems with an Adaptive Hierarchical Fracture Representation. PhD dissertation, ETH Zurich, Zurich (2013)

16. Karvounis, D., Gischig, V., Wiemer, S.: EGS probabalistic seismic hazard assessment with 3-D discrete fracture modeling. In: Proceedings of the Thirty-Ninth Workshop on Geothermal Reservoir Engineering, Stanford (2014)

17. Kazemi, H.: Pressure transient analysis of naturally fractured reservoirs with uniform fracture distribution. SPE J. 9(4), 451-462 (1969)

18. Kim, J., Tchelepi, H., Juanes, R.: Stability, accuracy, and efficiency of sequential methods for coupled flow and geomechanics. SPE J. 16(2), 249-262 (2011)

19. Lee, S.H., Jensen, C.L., Lough, M.F.: Efficient finite-difference model for flow in a reservoir with multiple length-scale variations. SPE J. 5(3), 268-275 (2000)

20. Lee, H.S., Cho, T.F.: Hydraulic characteristics of rough fractures in linear flow under normal and shear load. Rock Mech. Rock Eng. 35(4), 299-318 (2002)

21. Li, L., Lee, S.H.: Efficient field-scale simulation of black oil in a naturally fractured reservoir through discrete fracture networks and homogenized media. SPE Reserv. Eval. Eng. 11(4), 750-758 (2008) 
22. McClure, M.W., Horne, R.N.: Investigation of injection-induced seismicity using a coupled fluid flow and rate/state friction model. Geophysics 76(6), 181-198 (2011)

23. McClure, M.W.: Modeling and characterization of hydraulic stimulation and induced seismicity in geothermal and shale gas reservoirs. $\mathrm{PhD}$ dissertation, Stanford University, Stanford (2012)

24. McClure, M.W., Horne, R.N.: Discrete Fracture Network Modeling of Hydraulic Stimulation: Coupling Flow and Geomechanics. SpringerBriefs in Earth Sciences, Springer (2013)

25. Moinfar, A., Varavei, A., Sepehrnoori, K., Johns, R.T.: Development of a novel and computationally efficient discrete-fracture model to study IOR processes in naturally fractured reservoirs. Paper SPE 154246 presented at the Eighteenth SPE Improved Oil Recovery Symposium, Tulsa (2012)

26. Moinfar, A., Varavei, A., Sepehrnoori, K., Johns, R.T.: Development of a coupled dual continuum and discrete fracture model for the simulation of unconventional reservoirs. Paper SPE 163647 presented at the SPE Reservoir Simulation Symposium, The Woodlands (2013)

27. Norbeck, J., Huang, H., Podgorney, R., Horne, R.: An integrated discrete fracture model for description of dynamic behavior in fractured reservoirs. In: Proceedings of the 39th Workshop on Geothermal Reservoir Engineering, Stanford (2014)

28. Norbeck, J.H., Horne, R.N.: An embedded fracture modeling framework for fluid flow, geomechanics, and fracture propagation. In: Proceedings of the International Conference on Discrete Fracture Network Engineering, Vancouver, British Columbia, Canada (2014)

29. Norbeck, J., Horne, R.: Injection-triggered seismicity: An investigation of porothermoelastic effects using a rate-and-state earthquake model. In: Proceedings of the 40th Workshop on Geothermal Reservoir Engineering, Stanford (2015)

30. Olson, J.E.: Fracture aperture, length and pattern geometry development under biaxial loading: a numerical study with applications to natural, cross-jointed systems. In: Lewis, H., Couples, G.D. (eds.) The Relationship between Damage and Localization, pp. 123-142. Geological Society, London, Special Publications, The Geological Society of London (2007)
31. Peaceman, D.W.: Interpretation of well-block pressures in numerical reservoir simulation. SPE J. 23(3), 531-543 (1978)

32. Pluimers, S.: Hierarchical Fracture Modeling Approach. MSc thesis, Delft University of Technology, Delft (2015)

33. Rangarajan, R., Chiaramonte, M.M., Hunsweck, M.J., Shen, Y., Lew, A.J.: Simulating curvilinear crack propagation in two dimensions with universal meshes. Int. J. Numer. Meth. Eng. (2014)

34. Segall, P.: Earthquake and Volcano Deformation. Princeton University Press, Princeton (2010)

35. Shewchuk, J.R.: Triangle: Engineering a 2D quality mesh and delaunay triangulator. Lect. Notes Comput. Sci. 1148, 203-222 (1996)

36. Shiozawa, S., McClure, M.W.: EGS designs with horizontal wells, multiple stages, and proppant. In: Proceedings of the 39th Workshop on Geothermal Reservoir Engineering, Stanford (2014)

37. Shou, K.J., Crouch, S.L.: A higher order displacement discontinuity method for analysis of crack problems. Int. J. Rock Mech. Min. Sci. Geomech. Abstr. 32(1), 49-55 (1995)

38. Snow, D.T.: A parallel plate model of fractured permeable media. PhD dissertation, University of California, Berkeley (1965)

39. Valko, P., Economides, M.J.: Hydraulic Fracture Mechanics. Wiley, Chichester (1995)

40. Vermylen, J.P., Zoback, M.D.: Hydraulic fracturing, microseismic magnitudes, and stress evolution in the Barnett shale, Texas, USA. Paper SPE 140507 presented at the SPE Hydraulic Fracturing Technology Conference and Exhibition, The Woodlands (2011)

41. Vinsome, P.K., Westerveld, J.: A simple method for predicting cap and base rock heat losses in thermal reservoir simulators. J. Can. Pet. Tech. 19(3), 87-90 (1980)

42. Warren, J.E., Root, P.J.: The behavior of naturally fractured reservoirs. SPE J. 3(3), 245-255 (1963)

43. Willis-Richards, J., Watanbe, K., Takahashi, H.: Progress towards a stochastic rock mechanics model of engineered geothermal systems. J. Geophys. Res. 101(B8), 17481-17496 (1996)

44. Zoback, M.D.: Reservoir Geomechanics. Cambridge University Press, Cambridge (2007) 